\title{
Comment on "An Increase in Dietary Supplement Exposures Reported to US Poison Control Centers"
}

\author{
Richard L. Kingston ${ }^{1,2}$ • Andrea W. Wong ${ }^{3}$. Ikhlas Khan ${ }^{4}$
}

Received: 18 October 2017 / Accepted: 7 November 2017 / Published online: 28 November 2017

(C) The Author(s) 2017. This article is an open access publication

In the report by Rao et al., the authors' conclusions raise questions about the safety of dietary supplements (DS); however, there are important study limitations and context that must be considered before drawing conclusions regarding DS safety [1].

The current report purports to review exposures involving the FDA-regulated category of DS products. Yet, several product categories included in the analysis involved products clearly outside the scope of DS, including homeopathic products (representing 36\% of the dataset), which are regulated by the US Food and Drug Administration (FDA) as drugs, and beverage versions of energy drinks, which are regulated as foods. Cultural medicines typically include substances that are outside of any regulated category of product. Ma huang products, which accounted for numerous reported exposures, have not been on the market for over a decade and are not relevant to today's 170 million consumers who take DS as part of their wellness efforts [2].

For those calls that involve exposure to properly categorized DS, it must be emphasized that reports of exposure are not synonymous with adverse events. Parents

Richard L. Kingston

rkingston@safetycall.com

1 SafetyCall International, PLLC, 3600 American Boulevard W, Suite 725, Bloomington, MN 55431, USA

2 College of Pharmacy, University of Minnesota, Minneapolis, MN, USA

3 Scientific and Regulatory Affairs, Council for Responsible Nutrition, Washington, DC 20036, USA

4 School of Pharmacy, University of Mississippi, University, MS, USA calling for reassurance subsequent to childhood ingestion of non-toxic amounts of these and other household products are routinely represented in the most recent American Association of Poison Control Centers' (AAPCC) annual report in which $89 \%$ of all pediatric ( $<6$ years) exposures in the database involve no reports of adverse effects following exposure [3]. For the current report, asymptomatic exposures involve as many as $96 \%$ of all pediatric exposures and $99.6 \%$ when including minor outcomes.

There are also concerns with the manner in which medical outcomes were further categorized as "serious," which leads readers to equate those outcomes to life-threatening effects typically requiring life-saving therapeutic interventions, hospitalization, and potential residual disability. Yet, for this report, incidents coded with a moderate outcome were included in the "serious" outcome category. Moderate outcomes within the poison center dataset are, by definition, non-life threatening, leave no residual disability, and can include effects such as mild, self-limiting tachycardia, hives, or rash that require no therapeutic intervention. And, even for those serious events included in the PC data that involve allergy and/or anaphylaxis, confirmation that a DS was the cause with confirmatory allergy testing is rare. Removing the moderate outcome incidents from the report's serious outcome classification leaves only $0.2 \%$ as serious incidents as compared to $4.5 \%$ reported by the authors.

Equally concerning is the inclusion of incidents coded as death without some qualification or further review of the relatively small number of such events $(n=34)$. As an example, death associated with ginseng is highly suspect and cannot be independently confirmed, especially when fatality abstracts for this and virtually all reported fatalities presented in the dataset are missing both in this report and the corresponding AAPCC annual reports. 
Lastly, trends regarding absolute numbers of calls involving DS exposure are difficult to interpret without a denominator, a shortcoming that has plagued the analysis of poison center calls for decades [4]. Without a corresponding comparison to sales, including trends for symptomatic exposures over time, the analysis lacks critical context.

\section{Sources of Funding None.}

\section{Compliance with Ethical Standards}

Conflict of Interest Author Richard L. Kingston works for a firm providing safety consultation to dietary supplementcompanies. Author Ikhlas A. Khan declares he has no conflict of interest. Author Andrea W. Wong is employed by aprofessional trade association representing dietary supplement companies that receives funding through the dues ofits member companies.

Open Access This article is distributed under the terms of the Creative Commons Attribution 4.0 International License (http:// creativecommons.org/licenses/by/4.0/), which permits unrestricted use, distribution, and reproduction in any medium, provided you give appropriate credit to the original author(s) and the source, provide a link to the Creative Commons license, and indicate if changes were made.

\section{References}

1. Rao N, et al. 2017 An increase in dietary supplement exposures reported to US Poison Control Centers. J Med Toxicol. 13:227. https://doi.org/10.1007/s13181-017-0623-7

2. CRN, Council for Responsible Nutrition. 2016 CRN consumer survey on dietary supplements. Available at URL: http://www.crnusa. org/resources/crn-2016-annual-survey-dietary-supplements . Accessed 16 October 2017.

3. Mowry JB, Spyker DA, Brooks DE, Zimmerman A, Schauben JL. 2015 annual report of the American Association of Poison Control Centers' National Poison Data System (NPDS): 33rd annual report. Clin Toxicol (Phila). 2016;54(10):924-1109. https://doi.org/10. 1080/15563650.2016.1245421.

4. Hamilton RJ, Goldfrank LR. Poison center data and the Pollyanna phenomenon. J Toxicol Clin Toxicol. 1997;35(1):21-3. https://doi. org/10.3109/15563659709001160. 